\title{
An Improved MMC Control Strategy with Single-Phase to Ground Fault-Tolerance Capability
}

\author{
Huiyu Miao*, Jun Mei*, Jianyong Zheng ${ }^{\dagger}$, Chenyu Zhang**, Chao Zhu***, \\ Tian Ma**** and Fei Mei ${ }^{\S}$
}

\begin{abstract}
In this paper, an improved modular multilevel converter (MMC) control strategy based on one-cycle control (OCC) is proposed. With the introduction of OCC, the stability, rapidity and disturbance rejection of MMC is greatly improved. Based on the volt-second equilibrium, the control is distributed to every single submodule (SM). For further stability, an unbalanced OCC strategy is designed based on feed-forward control for negative sequence voltage with no need for measuring the positive and negative current sequence, considering single phase to ground fault and the second harmonic ripples of the DC voltage. As the switch frequency of every SM is different, the virtual loop mapping is used in this paper to balance the capacitor voltage with less hardware dependence. And a new simplified structure is also proposed with RS flip-flop abandoned. At last, simulation and experiment results prove the validity of the control theory.
\end{abstract}

Keywords: MMC, OCC, Single phase to ground fault, Capacitor balance

\section{Introduction}

Modular multilevel converter (MMC) is a new kind of converter which can be used in medium- and high-voltage applications, especially in high voltage direct current (HVDC) [1-2]. MMC has the great features compared with the traditional converters, e.g. lower total harmonic distortion, high availability and modularity, lower switch voltage and so on [3-4]. The single submodule (SM) of MMC can be controlled and extended easily with simple structure [5]. However, the cost and the control complexity increase linearly with the extension of SMs.

Control strategy for MMC has become the research hotspot. It contains the modulation of inverter, the capacitor voltage balance, operation control strategy and the circulating current suppression etc. In [6], space vector PWM can be commonly used to control MMC with appropriate resultant sequence. The calculation and space vectors become extremely complex with the increase number of SM. The phase-shifted carrier PWM (PSCPWM) is applied in MMC in [7] and the number of carrier wave is same with the SMs in one arm. The switching angles of every electrical level step are obtained based on area equivalent principle in [8], and the output voltage is modulated. Multilevel harmonic elimination technology is introduced to MMC in order to suppress harmonics and

\footnotetext{
$\dagger$ Corresponding Author: School of Electrical Engineering, Southeast University, China.

* School of Electrical Engineering, Southeast University, China.

** Jiangsu Electric Power Research Institute, China.

*** Jiangsu Electric Power Maintenance Branch Company, China.

****State Grid Jiangsu Economic Research Institute, China.

$\S \quad$ College of Energy and Electrical Engineering, Hohai University, China.
}

Received: September 24, 2015; Accepted: April 14, 2016 decrease the switching frequency in [9]. Reference [10] maintains the balance of capacitor voltage by switching SMs in specific order of voltage. The calculation and measurement units increases with extension. Reference [11] gives detailed analysis on the combination of PWM and the capacitor voltage balance. The capacitor voltage is introduced as the control signals to modulation wave in [12] and the sequencing process of voltage can be cancelled. However, measurement units of every single SM cannot be abandoned. Reference [13] proposes a method to maintain the capacitor voltage with lower switch frequency. Overall, the control strategy has relationships with the extension of SMs and the structure of MMC. The accuracy, rapidity and stability is affected by the redundant structure and complex control.

Besides the condition of normal operation, fault ridethrough capability is also very important which affects the safety and the stability of the grid and MMC. The unbalanced situation and the single-phase ground fault often occur in the progress of actual operation of power grid. There are second harmonics in the voltage of DC side and distortion in the grid-connected current [14].

There are already many researches on the unbalanced control. The first study on dynamic system under unbalanced condition is in [15] with a back-to-back MMC structure. Reference [16] introduces a dedicated DC voltage ripple suppressing controller based on the instantaneous power. Reference [17] uses proportional resonant (PR) controller to keep the current tracking floating. Reference [18] proposes a SR controller to suppress the second harmonics in the DC side. But the PR controller is relatively complex to design. Reference [19] proposes an unbalanced control based on OCC with current controlled 
parameter to amend the control. But there is singular point in the parameter when positive and negative sequence is the same and the control accuracy is affected.

One cycle control (OCC) is proposed by Keyue M. Smedley and Slobodan Cuk[20]. It is a new kind nonlinear control method which control the time-varying voltage and current instantaneously. The dynamic response speed is fast and the input signal disturbance is restrained. The dynamic response speed is fast and the input signal disturbance is restrained. OCC tracks the reference waveform accurately and eliminates the integral error in one cycle with strong robustness. In [21], OCC is better than PWM control in reducing undesirable harmonics and tracing dynamic waveforms with application in power amplifier of multilevel converter. OCC is extended to control the three-phase active power filter with similarity and solid stability in [22]. Reference [23] proposes OCC for double-input buck converter to simplify the control. The two control of loops are independent of each other.

In this paper, one-cycle control (OCC) is applied in the control of MMC. OCC is composed of resetting integrator and some simple linear logic devices which can simplify the control strategy of MMC with only one PI controller needed. By volt-second equilibrium, the basic control is distributed to every single SM. The relationship between the virtual switching function of the whole model and the switch function of single SM is established. The basic idea is to make the switch variables of every SM equal to the control signal by changing the duty cycle of the switch. As the control to every single part only contains some simple logic devices, the control strategy is greatly simplified. The response speed, accuracy and the disturbance of MMC is improved because the control signal of SM is generated in each clock cycle which can be set according to the specific operating conditions. For further stability and single phase to ground fault-tolerance capability, the improved control strategy is introduced in this paper. By feed-forward control for negative sequence voltage, MMC works on the condition of constant power. With no need for measuring the positive and negative current sequence, the whole control is simplified and the rapidity is increased. As the switch frequencies of SMs based on control strategy above is different, the voltage of capacitor is definitely unbalanced. In order to solve the problem, the virtual loop mapping is introduced in the paper. By specific map relationship, the voltage is balanced with no need to measure the SM and further calculation. The conventional OCC structure is also optimized and the RS flip-flop is abandoned. And the whole scheme of the control only need one PI controller with the improved structure.

\section{The Mathematical Model of MMC}

Fig. 1 shows the topology of three-phase MMC and the structure of submodule (SM). The MMC works in the case

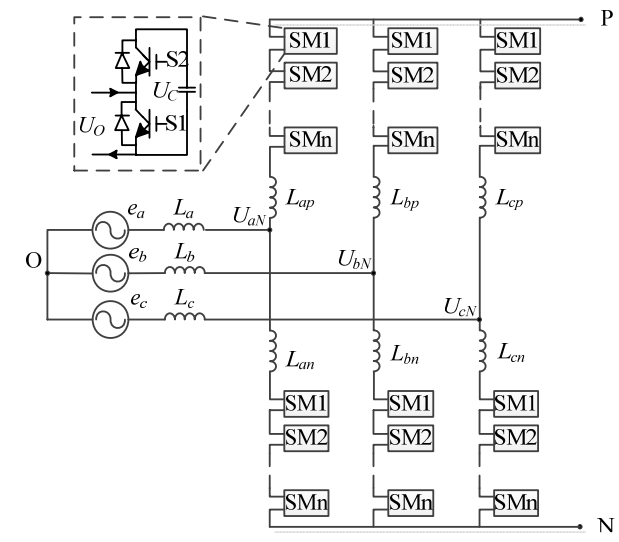

Fig. 1. The topology of three-phase MMC

as an inverter. The SM contains a flying-capacitor and the IGBT half-bridge. The output of the SM is changed between $U_{C}$ and 0 , control by the switch state of the IGBT $\mathrm{S} 1$ and $\mathrm{S} 2$. The output of one SM can also be expressed as

$$
U_{O}=d_{n} \cdot U_{C}
$$

In (1), $U_{O}$ is the output of the SM. $U_{C}$ is the voltage of the fly-capacitor and $d_{n}$ is the switch function of the SM.

$L_{x p}$ and $L_{x n}(x=\mathrm{a}, \mathrm{b}, \mathrm{c})$ are the inductors in the upper and lower arms, which are used to suppress the unbalance current and the circulating current. $U_{a N}, U_{b N}, U_{c N}$ are output voltages of each leg. $L_{a}, L_{b}, L_{c}$ are the grid-side filter inductors. $e_{a}, e_{b}, e_{c}$ are grid voltages. $\mathrm{O}$ is the neutral point of the three phase network. $\mathrm{P}$ and $\mathrm{N}$ are the positive and negative sides of DC.

The SMs in the upper and lower arms can be equivalent as controlled voltage sources $U_{p j}$ and $U_{n j}$. According to the Kirchhof voltage law, the output follow the relationship as [24]

$$
\left\{\begin{array}{l}
U_{x N}=V_{d c}-U_{p j}-L_{x} \frac{d i_{p j}}{d t} \\
U_{x N}=-V_{d c}+U_{n j}+L_{x} \frac{d i_{n j}}{d t} \\
U_{x N}=\frac{U_{n j}-U_{p j}}{2}-\frac{L_{x}}{2} \cdot \frac{d i_{x}}{d t}
\end{array}\right.
$$

In (2), $x=\mathrm{a}, \mathrm{b}, \mathrm{c}$, which mean the three phases. $i_{p j}$ and $i_{n j}$ are the current in the upper and lower arm. $i_{x}$ is output current of one leg. The model of MMC is similar to the two-level VSC. Many control strategies for VSC, e.g., the inner current control loop and the outer active power control loop, can be applied in the control of MMC.

\section{MMC Control Strategy Based on OCC}

\subsection{Overall mathematical model}

The average output voltage of MMC can be set as (3), 
which is the function of the voltage in the DC side. $D_{x}$ is the virtual switching function of the whole model. $x=\mathrm{a}, \mathrm{b}, \mathrm{c}$.

$$
U_{x N}=D_{x} \cdot V_{d c}
$$

If the inductance between MMC and the grid is small enough, the voltage drop over it can be ignored. So the voltage $U_{x O}$ of nodes $\mathrm{A} \mathrm{B} \mathrm{C}$ in the $\mathrm{AC}$ side relative to neutral point $\mathrm{O}$ satisfies the following relationship.

$$
e_{x}=U_{x O}=U_{x N}+U_{N O}
$$

In (4), $U_{N O}$ is the voltage difference between the $\mathrm{O}$ and the negative DC side. The zero sequence current can be ignored as three-phase three-wire system is adopted. (4) can be further derived as

$$
\left\{\begin{array}{l}
U_{A O}+U_{B O}+U_{C O}=0 \\
U_{N O}=-\frac{1}{3}\left(U_{\mathrm{AN}}+U_{\mathrm{BN}}+U_{\mathrm{CN}}\right)
\end{array}\right.
$$

By substituting (5) and (2) to (4), the DC and the grid voltage have the relationship as [25]

$$
\left[\begin{array}{ccr}
\frac{2}{3} & -\frac{1}{3} & -\frac{1}{3} \\
-\frac{1}{3} & \frac{2}{3} & -\frac{1}{3} \\
-\frac{1}{3} & -\frac{1}{3} & \frac{2}{3}
\end{array}\right]\left[\begin{array}{l}
D_{A} \\
D_{B} \\
D_{C}
\end{array}\right] \cdot V_{\mathrm{dc}}=\left[\begin{array}{l}
e_{a} \\
e_{b} \\
e_{c}
\end{array}\right]
$$

The matrix rank in (6) is 2 , so the solution can be as

$$
\left\{\begin{array}{l}
D_{A}=k_{1}+e_{a} / V_{d c} \\
D_{B}=k_{1}+e_{b} / V_{d c} \\
D_{C}=k_{1}+e_{c} / V_{d c}
\end{array}\right.
$$

In (7), $k_{1}$ can be any number within the appropriate range, which is not changed by the instantaneous value of the grid voltage. In this paper, $k_{l}$ is set to 0.5 .

As the MMC work in the condition of unity power factor, the current and voltage of the grid side follow the relationship as

$$
i_{x}=e_{x} / R_{\mathrm{e}}
$$

In (8), $R_{e}$ is the equivalent power resistors [26]. With the introduction of $k_{2}$ and $k_{3}$, (8) can be changed as

$$
\left\{\begin{array}{l}
i_{x}=k_{3} e_{x}-k_{2} e_{x} \\
k_{3}-k_{2}=1 / R_{\mathrm{e}}
\end{array}\right.
$$

The parameter $k_{3}$ is used to restrict the grid current. And $k_{2}$ is used to control the power [26]. With introduction of resistance $R_{s}$ to sample the current, the further derivation of (9) is as

$$
\left\{\begin{array}{l}
R_{s} i_{x}=k_{3} R_{s} e_{x}-k_{2} R_{s} V_{d c} e_{x} / V_{d c} \\
k_{3}-k_{2}=1 / R_{\mathrm{e}}
\end{array}\right.
$$

Substitute (7) to (10), then

$$
R_{s} i_{x}=k e_{x}-V_{m}\left(\frac{D_{x}}{k_{1}}-1\right)
$$

And

$$
\left\{\begin{array}{l}
k=k_{3} \cdot R_{s} \\
V_{m}=\left(k-\frac{R_{\mathrm{s}}}{R_{\mathrm{e}}}\right) k_{1} V_{\mathrm{dc}}
\end{array}\right.
$$

In (11), $k$ is used to restrict the grid current, which is similar to $k_{3} . D_{x}$, the virtual switching function, is related to the integration time constant of the integrator in the one cycle control system. $V_{m}$ is the controlling voltage of error with the reference in DC side in one cycle control. Similar to $k_{2}$, it can control the power of MMC. PI controller can be used to hold the voltage in the DC side, and the control equation is as

$$
V_{m}=\left(K_{v P}+K_{v I} \int d t\right)\left(V_{\mathrm{dc}}^{*}-V_{d c}\right)
$$

$K_{v P}$ and $K_{v I}$ are the proportional and the integral control factor of the PI controller of the DC side. $V_{d c}{ }^{*}$ is the reference voltage of the DC side. $V_{d c}$ is the actual value of the DC voltage.

Fig. 2 shows the logic diagram of the overall control based on OCC under normal condition. According to (13), $V_{m}$ is the output of the PI controller after the difference between $V_{d c}$ and the reference. In one cycle, $V_{m}$ is integrated and multiplied by the coefficient $m$, which is on the basis of (11), and then the result is compared with the parameters. At last, the comparative result enters the RS flip-flop with the clock signal and the output is the control signals. $\bar{Q}$ is also used to reset the integrator.

\subsection{SM Mathematical Model}

The overall mathematical model is established as (11),

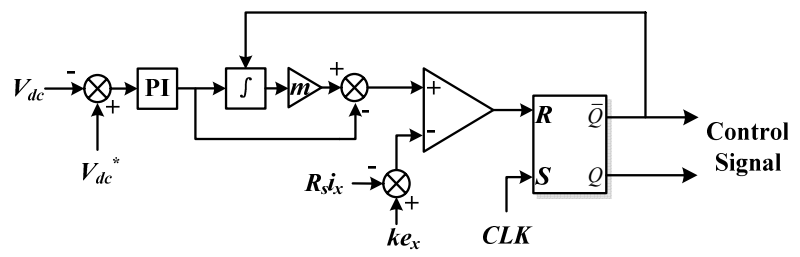

Fig. 2. The control under normal condition 
Table1. The states of SM and output regions

\begin{tabular}{|c|c|c|c|c|c|c|c|c|c|}
\hline \multirow{2}{*}{ State } & \multicolumn{4}{|c|}{ upper arm } & \multicolumn{4}{|c|}{ lower arm } & \multirow{2}{*}{$\begin{array}{l}\text { Output } \\
\text { voltage }\end{array}$} \\
\hline & 1 ' & 2, & $3^{\prime}$ & 4 & 5 , & 6, & 7 ' & 8 ' & \\
\hline I & PWM & 0 & 0 & 0 & PWM & 1 & 1 & 1 & $2 U_{C} \sim U_{C}$ \\
\hline II & 1 & PWM & 0 & 0 & 0 & PWM & 1 & 1 & $U_{C} \sim 0$ \\
\hline III & 1 & 1 & PWM & 0 & 0 & 0 & PWM & 1 & $-U_{C} \sim 0$ \\
\hline IV & 1 & 1 & 1 & PWM & 0 & 0 & 0 & PWM & $-U_{C \sim-2 U_{C}}$ \\
\hline
\end{tabular}

$D_{x}$ is the virtual switching function of the whole model. However the control have to concrete to state of SM. The overall MMC switch function have to distribute to every single switch in one SM.

In order to analysis the model of MMC more conveniently, it is assumed that there are totally $8 \mathrm{SMs}$ in one leg. In this case, the output state of MMC can be divided into 4 regions according to the state of SMs (on, off and PWM state), shown in Table 1.

In Table 1, 1' 8' are the SM of the MMC. 1' $\sim 4$ ' are the SMs in the upper arm and 5' 8' are the SMs in the lower arm. " 1 " means the switch is on and the SM is cut-in. " 0 " meads the switch is off and the SM is cut-off. PWM means the switch of the SM keeps changing between on and off, and the PWM signal varies from " 1 " to " 0 ", which is corresponds to the duty cycle. The signal of the upper arm is contrary to the lower arm. $U_{C}$ is the voltage of the flyingcapacitor in a single SM. In every region, the output can be derived as (14) according to (1) and the volt-second equilibrium.

$$
\left\{\begin{array}{l}
u_{1}=d_{1} U_{C}=d_{1} V_{d c} / 4 \\
u_{2}=d_{2} U_{C}+U_{C}=d_{2} V_{d c} / 4+V_{d c} / 4 \\
u_{3}=d_{3} U_{C}+2 U_{C}=d_{3} V_{d c} / 4+V_{d c} / 2 \\
u_{4}=d_{4} U_{C}+3 U_{C}=d_{4} V_{d c} / 4+3 V_{d c} / 4
\end{array}\right.
$$

In (14), $d_{X}(x=1,2,3,4)$ is switch function of the SM which is in the PWM state. Comparing with (1), the overall equivalent switch function can be changed as

$$
\left\{\begin{array}{l}
D=d_{1} / 4 \\
D=d_{2} / 4+1 / 4 \\
D=d_{3} / 4+1 / 2 \\
D=d_{4} / 4+3 / 4
\end{array}\right.
$$

Substitute (15) to (11), the control of every single SM is as

$$
\left\{\begin{array}{l}
R_{s} i_{x}=k e_{x}-V_{m}\left(d_{1} / 4 k_{1}-1\right) \\
R_{s} i_{x}=k e_{x}-V_{m}\left[\left(d_{2}+1\right) / 4 k_{1}-1\right] \\
R_{s} i_{x}=k e_{x}-V_{m}\left[\left(d_{3}+2\right) / 4 k_{1}-1\right] \\
R_{s} i_{x}=k e_{x}-V_{m}\left[\left(d_{4}+3\right) / 4 k_{1}-1\right]
\end{array}\right.
$$

and

$$
d_{x}=t_{x} / T_{S}
$$

In (17), $t_{x}$ is the turn-on time of SM, And $T_{S}$ is the switching cycle which is relevant to the integration time constant of the integrator in the one cycle control system.

The control strategy is based on one phase, and the other two phases can be derived in a similar way. The integration variable of one SM keeps comparing with the reference of the one cycle control system in one clock cycle, if the variable is smaller and the switch keeps on.

\section{Single-Phase to Ground Fault-Tolerance Capability}

The control strategy above is based on the condition of normal operation. When the grid break down, e.g., singlephase grounding, the system stability and operation are affected. There is negative-sequence current in the AC side when accident happens, and the protection of the system operation unwanted. The negative-sequence voltage and current in the AC side also cause the power fluctuation during the transmission. The fluctuation lead to the voltage ripples in the DC side. By DC network, the ripples transmit to the other parts of the whole system and cause the instability and insecurity of the whole system.

In order to suppress the ripples, an advanced control strategy based on the theory above is introduced as below. The zero-sequence voltage of the grid has no effect on the voltage in the DC side as the a three-phase three-wire system is adopted, so emphasis is laid on the other components. The grid voltage can be resolved into

$$
e_{x}^{\prime}=e_{x}-\left(e_{a}+e_{b}+e_{c}\right) / 3=e_{x+}+e_{x-}
$$

When the grid voltage is unbalanced, the active power need to keep constant in order to suppress the ripples. The positive sequence and negative sequence of the grid voltage and current have to follow the relationship as [27]

$$
\left\{\begin{array}{l}
{\left[\varphi_{+}(t)-\theta_{+}(t)\right]+\left[\varphi_{-}(t)-\theta_{-}(t)\right]=180^{\circ}} \\
\frac{I_{+}}{I_{-}}=\frac{E_{+}}{E_{-}}
\end{array}\right.
$$

In (19), $\varphi_{+}(t)$ and $\varphi_{-}(t)$ are the phases of the positive sequence and negative sequence voltage. $\theta_{+}(t)$ and $\theta_{-}(t)$ are the phases of the positive sequence and negative sequence current.

When MMC works on the condition of unit power factor, the phases of the positive sequence current have to be the same with the positive sequence voltage. And

$$
\varphi_{+}(t)=\theta_{+}(t)
$$

Considering (19), the phases of the negative sequence 
current and voltage follow the relationship as

$$
\varphi_{-}(t)-\theta_{-}(t)=180^{\circ}
$$

The components of voltage and current meet the following equation

$$
\left\{\begin{array}{l}
e_{x+}=R_{e+} i_{x+} \\
e_{x-}=R_{e-} i_{x-}
\end{array}\right.
$$

$R_{e^{+}}$and $R_{e_{-}}$are the equivalent positive sequence and negative sequence resistance. It can be assumed as

$$
R_{e}=R_{e+}=-R_{e-}
$$

And (18) can be further derived as

$$
e_{x}^{\prime}=e_{x+}+e_{x-}=R_{e+} i_{x+}+R_{e-} i_{x-}=R_{e} i_{x}+2 e_{x-}
$$

Formula (24) can be transferred as

$$
i_{x}+2 \frac{1}{R_{e}} e_{x-}=\frac{1}{R_{e}} e_{x}^{\prime}
$$

According to the OCC control strategy proposed earlier in this paper, with the introduction of resistance $R_{s}$ to sample the current and constant $k_{3}, k_{2},(25)$ can be transferred as

$$
\left\{\begin{array}{l}
-R_{s} i_{x}+V^{\prime}=V_{m}^{\prime}\left(\frac{D_{x}}{k_{1}}-1-\frac{2 e_{x-}}{k_{1} V_{d c}}\right) \\
V^{\prime}=k_{3} R_{s}\left(e_{x}-2 e_{x-}\right) \\
V_{m}^{\prime}=\left(k-\frac{R_{s}}{R_{e}}\right) k_{1} V_{d c}
\end{array}\right.
$$

Substitute (15) to (26), the control of every single SM is as

$$
\left\{\begin{array}{l}
-R_{s} i_{x}+V^{\prime}=V_{m}^{\prime}\left(d_{1} / 4 k_{1}-1-\frac{2 e_{x-}}{k_{1} V_{d c}}\right) \\
-R_{s} i_{x}+V^{\prime}=V_{m}^{\prime}\left[\left(d_{2}+1\right) / 4 k_{1}-1-\frac{2 e_{x-}}{k_{1} V_{d c}}\right] \\
-R_{s} i_{x}+V^{\prime}=V_{m}^{\prime}\left[\left(d_{3}+2\right) / 4 k_{1}-1-\frac{2 e_{x-}}{k_{1} V_{d c}}\right] \\
-R_{s} i_{x}+V^{\prime}=V_{m}^{\prime}\left[\left(d_{4}+3\right) / 4 k_{1}-1-\frac{2 e_{x-}}{k_{1} V_{d c}}\right]
\end{array}\right.
$$

$V_{m}$ ' is used to control the voltage error of DC side, which is similar to $V_{m}$. Only one PI regulator need be applied to stabilize voltage.

When the grid is unbalanced, there are positive and negative sequence voltage at the same time. The voltage of the DC side cannot be held by the control strategy (16), and there are distortion in the AC current. (27) adds the feed forward compensation component of the negative sequence voltage based on (16) and the MMC achieve the single phase to ground fault-tolerance capability.

\section{Capacitor Voltage Balance}

By using the control in the section above, the output is divided into several regions, and the switch frequency of each SM is different, which definitely cause the flyingcapacitor voltage unbalanced. In order to keep the unbalance voltage within certain range, the Virtual Loop Mapping (VLM) is introduced in the paper.

The main idea of VLM is that the PWM control signals of SMs is set as the virtual modules and the virtual ones map to the real ones in certain ways to keep the switch frequency approximately equal in every loop [28]. For example, there are $8 \mathrm{SMs}$ in one leg and the mapping is also divided into 4 states. Each state continues for one switch clock cycle. The mapping relationship is as Fig. 3. 1 ' -8 ' are the virtual modules. 1'-4' are in the upper bridge arm and 5'-8' are in the lower bridge arm.1-8 are the real ones, 1-4 in the upper and 5-8 in the lower. The arrows mean the mapping relationship. The 4 states constitute one loop. And the PWM control signals are distributed to the real modules averagely in one loop. From the point of one loop (4 states), the switch frequency of every SM is the same. In theory, the voltage of the flying-capacitor should

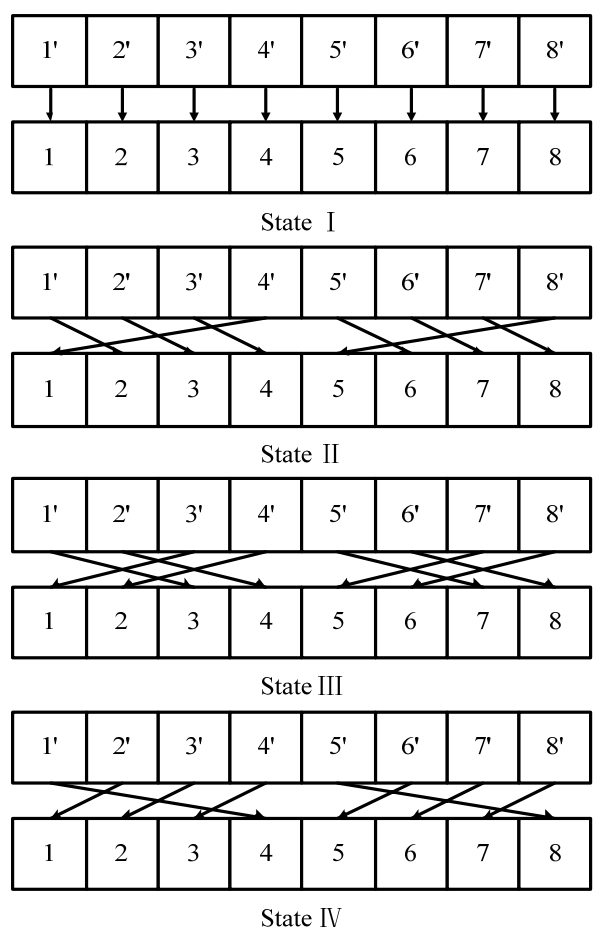

Fig. 3. The theory of the virtual loop mapping 
keep similar in one loop.

\section{The Improved Scheme of the Control System}

The traditional method to generate PWM signals in OCC is as Fig. 4 [29]. The controlled variables are processed by an integrator, and then compare with the reference. The result is processed through the RS flip-flop under the clock frequency and the PWM signals are generated at last. The signal $\bar{Q}$ is also used to reset the integrator. There is no problem in the theory and the simulation prove the correctness of the control method. However, in the experiment the control signals are interfered and the stability of the control strategy is affected. There are also narrow pulse signals affecting the reset of the integrator. It is because of the high switch frequency and the frequent status switching of the RS flip-flop.

The control strategy has to follow the practical experiment fact. In this paper, the RS flip-flop is abandoned and a simplify strategy is introduced, shown as Fig. 5.

The clock signal is used to reset the integrator every clock cycle. The controlled variables integrate in a whole clock period and reset to 0 before the next cycle. Compare the result with the reference and output is the PWM control signal. The improved circuit structure replaces the PR flipflop with the not gate. The output PWM signal become more stable and the effect of the narrow pulse on the reset signals is eliminated. Besides that, in the traditional structure, every single SM need one individual PI controller as the integrator reset signal is different by the PWM signals.

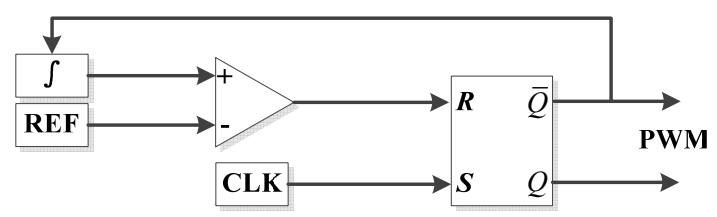

Fig. 4. The traditional structure of PWM generator

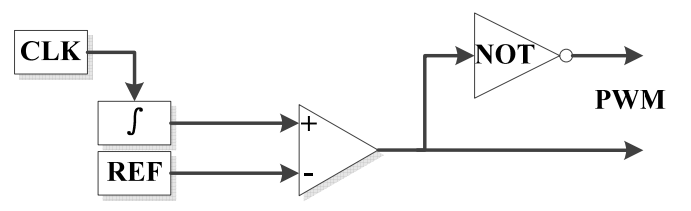

Fig. 5. The improved structure of PWM generator

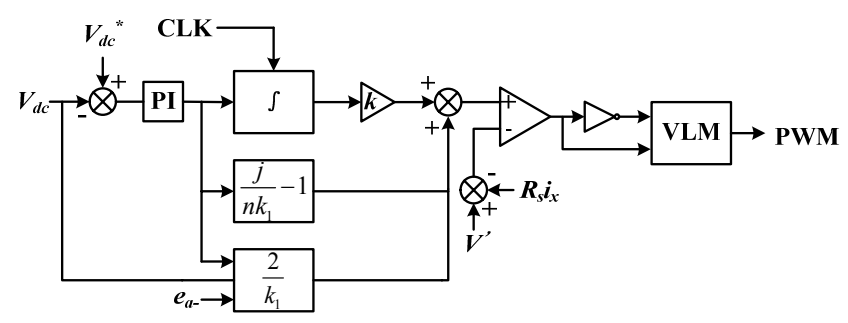

Fig. 6. The control strategy for SM
With the improved structure, only one PI controller is need in the over control scheme and the control is greatly simplified.

To sum up, the control strategy of the whole article is as follows. Fig. 6 shows the control for each SM. $n$ is number of SMs in the arm. And $j=0,1,2, \ldots$ n. $V_{m}$ ' is the output of the PI controller, used to control the voltage error of DC side. And $V_{m}$ ' is integrated in one cycle according to the clock signal. In the beginning of one cycle, the integrator is reset to 0 . The output of the integrator is calculated with the parameters on the basis of (25). The result compares with the reference. By the virtual loop mapping, the control signal is distributed to every single submodule.

\section{Simulation and Experiment Result}

To verify the feasibility and validity of OCC control strategy used in MMC, a system model as Fig.1 is established in Matlab/Simulink. Each arm has four SMs $(\mathrm{N}=4)$. The grid-connected transformer wiring is set as $\mathrm{Yn} / \Delta$ with the ration of $380 / 50$. The simulation parameters are shown in Table 2

In the simulation, it is assumed that single phase to ground fault happened at the time of $0.8 \mathrm{~s}$ and the advanced control method is added in the system at the time of $1.3 \mathrm{~s}$. In the PI controller, proportional coefficient is set to 0.1 , and integral coefficient is set to 50 . The clock frequency is set to $2000 \mathrm{~Hz}$. The system parameter $k$ is concerned with ratio of the $\mathrm{AC}$ voltage sensor and the gain of the conditioning circuit. $k$ is set to 0.5 and $R_{s}$ is the equivalent current sampling resistance which is set to $0.5 \Omega$. So $k_{3}$ has to be set to 1 . The simulation results are as below.

Fig. 7(a) shows the grid-connected current of three phase when the grid operates under normal condition. In Fig. 7(b), the basic control strategy based OCC works well and the DC voltage holds $150 \mathrm{~V}$. In Fig. 7(c), one single phase to ground fault happens and there is current distortion. Fig. 7(d) shows the DC side voltage at the same time. The peakto-peak value of the second harmonics in the DC side voltage is up to $3 \mathrm{~V}$ which accounts for $2 \%$ of the reference. Fig. 7(e) shows the grid-connected current when the advanced control method is added. In Fig. 7(f), the peakto-peak value of the second harmonics in the DC side voltage is suppressed to $1.5 \mathrm{~V}$ which accounts for $1 \%$ of the

Table 2. The simulation parameters

\begin{tabular}{c|c}
\hline Parameters & Values \\
\hline Number of SM in Each Arm & 4 \\
\hline Submodule Capacitor & $4700 \mathrm{uF}$ \\
\hline Arm Equivalent Resistance & $1.2 \mathrm{mH}$ \\
\hline AC Link Inductor $L_{x}$ & $1 \mathrm{mH}$ \\
\hline DC Bus Voltage $U_{d c}$ & $150 \mathrm{~V}$ \\
\hline AC System Line Voltage RMS & $50 \mathrm{~V}$ \\
\hline Power Frequency & $50 \mathrm{~Hz}$ \\
\hline$K_{v P}$ & 0.1 \\
\hline$K_{v I}$ & 50
\end{tabular}




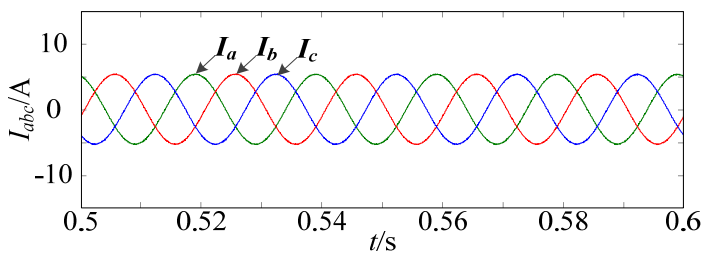

(a) AC currents

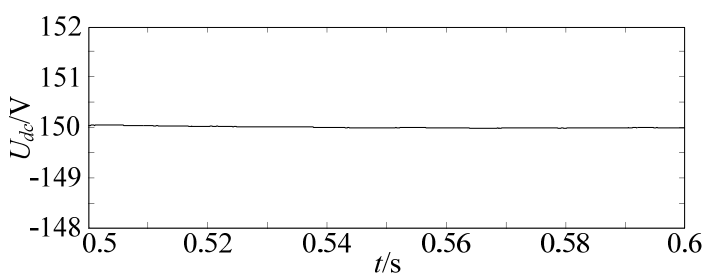

(b) DC bus voltage

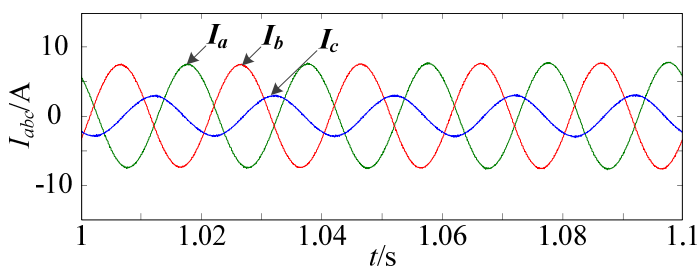

(c) AC currents with single phase to ground fault

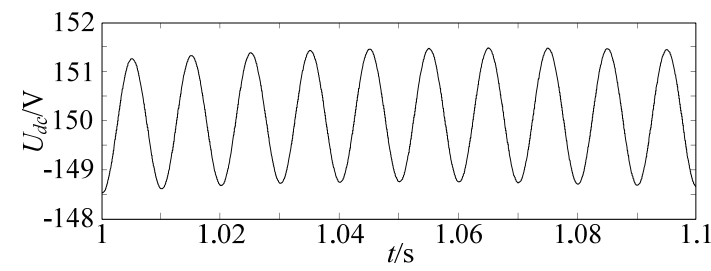

(d) DC bus voltage with single phase to ground fault

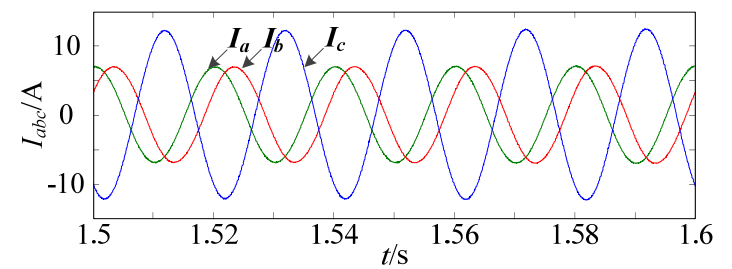

(e) AC currents with single phase to ground fault-tolerance capability

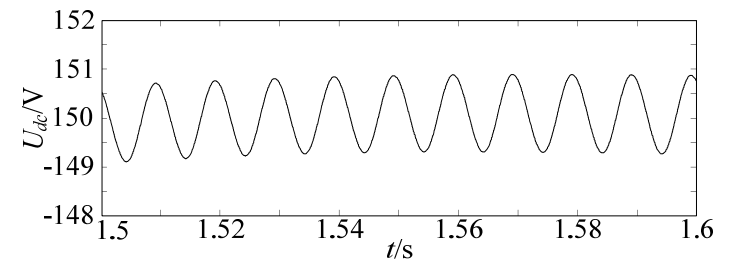

(f) DC bus voltage with single phase to ground faulttolerance capability

Fig. 7. Simulation diagrams of AC currents and DC bus voltage

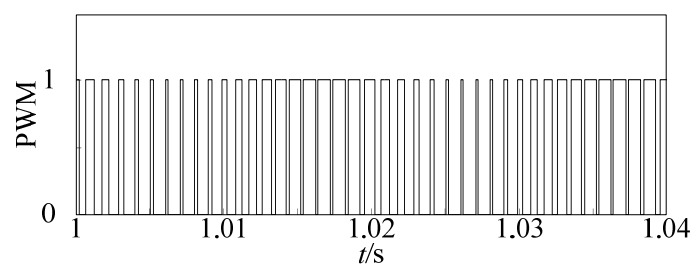

(a) PWM singles of SM

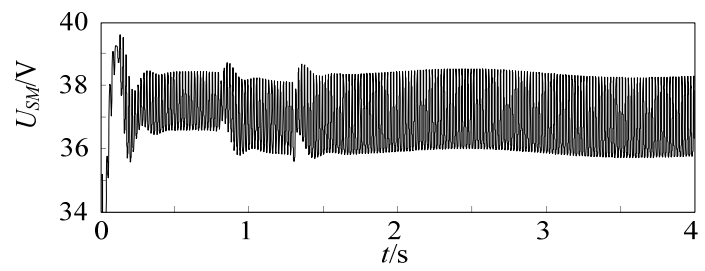

(b) The voltage of the capacitor in SM

Fig. 8. Simulation diagrams of SM

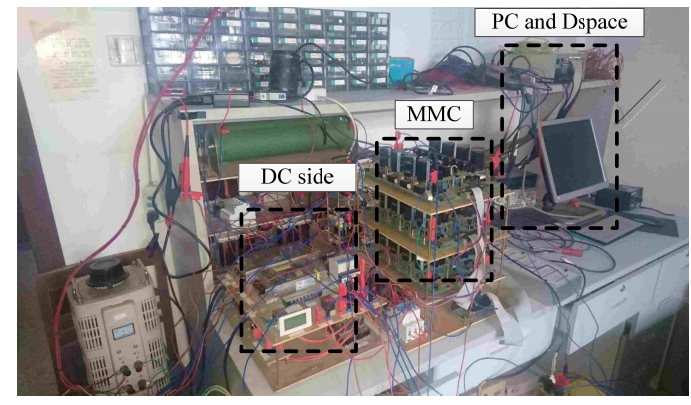

Fig. 9. The experimental set up

reference.

Fig. 8(a) is the PWM signal of the one SM of phase A in the upper arm. The signal is distributed relatively evenly over time. Fig. 8(b) is the capacitor voltage of the SM. With the introduction of the virtual loop mapping, the capacitor voltage is restricted to certain range.

The experiment is based on the platform of dSPACE DS1103 and the parameters are the same with simulation. Fig. 9 shows the experimental set up in the laboratory. As the circuit is a DC/AC converter essentially, the initial voltage of submodules in MMC is established by increase the voltage in the DC side smoothly with the grid off. The precharging process continues until the SM voltage meet the rated value.

In Fig. 10(a), the MMC operates on the normal condition and the grid is in the three-phase balance. Channel 1 is the voltage in the DC side and it holds $150 \mathrm{~V}$. And the gridconnected current and the grid voltage are also shown in Fig. 10(a). Fig. 10(b) shows the output voltage of MMC. Fig.11. shows the power and power factor of phase. Fig. 12. shows the current harmonics of phase A under normal operation. 


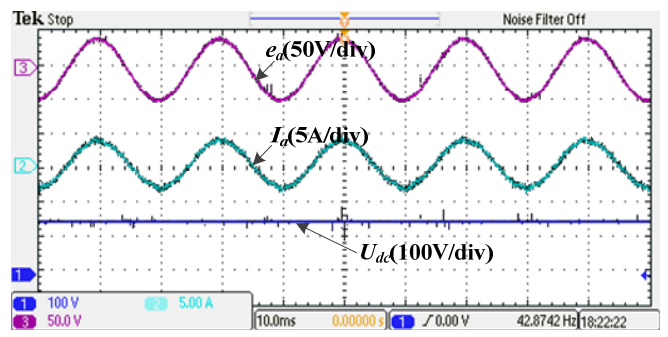

(a) Gird voltage, AC current and DC bus voltage

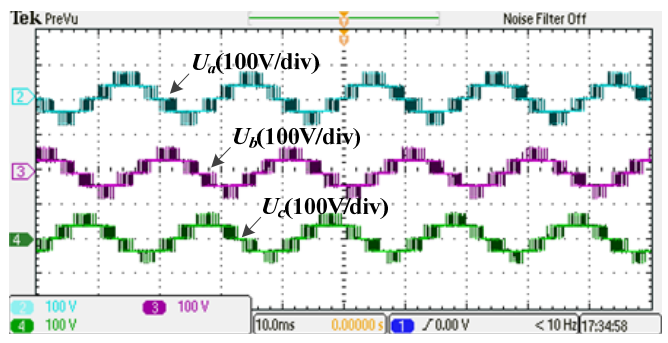

(b) Output voltage of MMC

Fig. 10. Experiment diagrams with on fault in grid

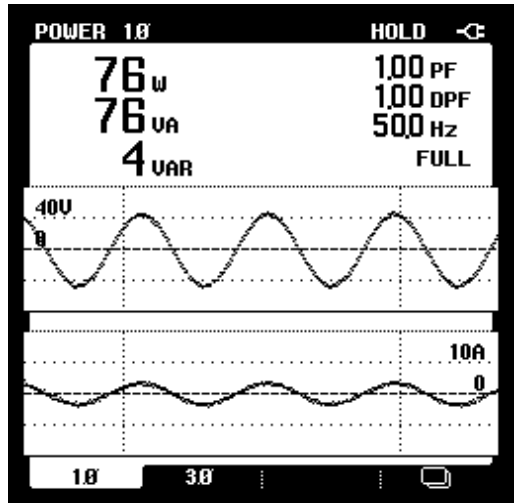

Fig.11. Power quality of phase A

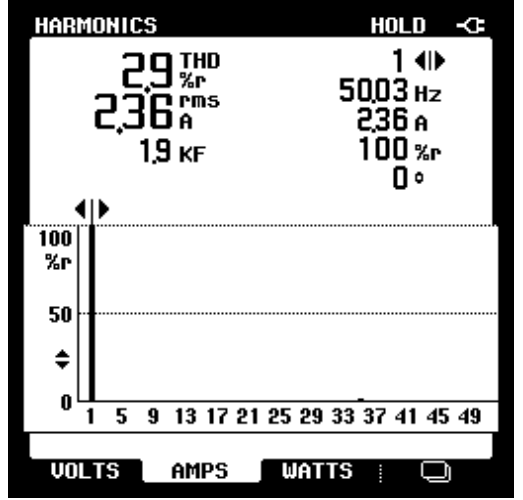

Fig. 12.Current harmonics of phase A

Fig. 13 shows the PWM signal of SM with improved circuit configuration and the capacity voltage of the same SM. The capacitor voltage of the SM holds around 37V, which prove the validity of the virtual loop mapping.

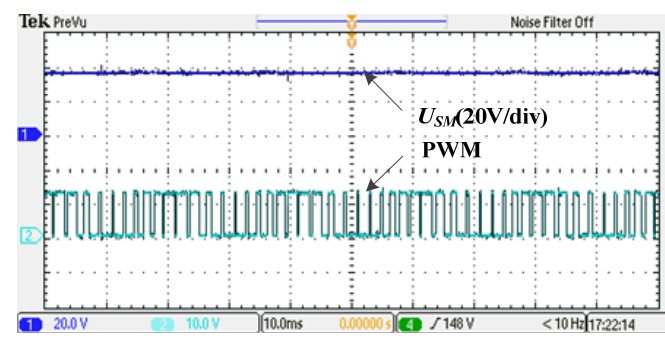

Fig. 13. Experiment diagrams of SM

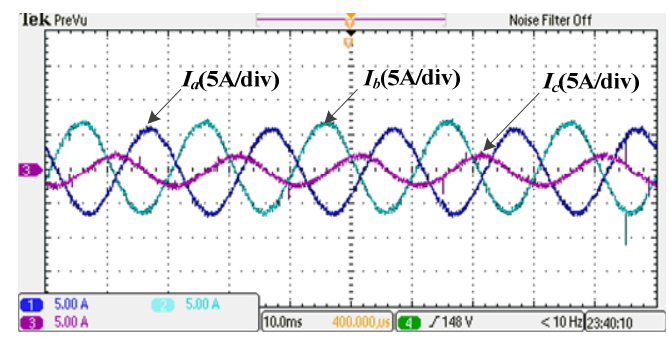

(a) AC currents with single phase to ground fault

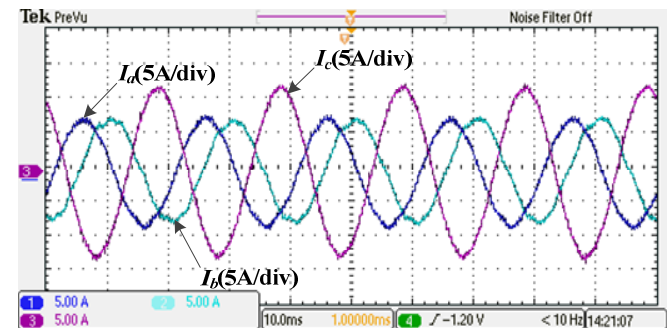

(b) AC currents with single phase to ground fault-tolerance capability

Fig. 14. Experiment diagrams of AC currents

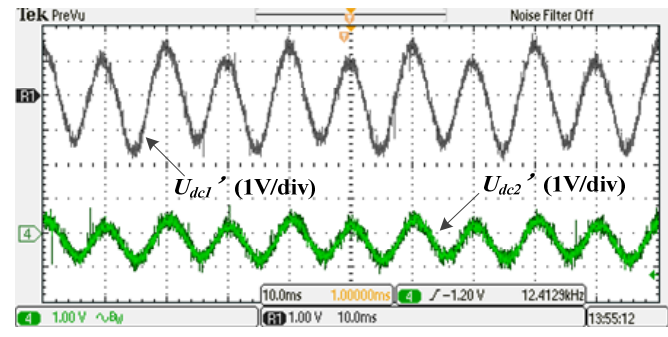

Fig. 15. Experiment diagram of DC bus voltage

When single phase to ground fault happens, (phase $\mathrm{C}$ is grounded in the experiment), the grid-connected current is shown in Fig. 14(a). The current in phase $\mathrm{C}$ is smaller than the current in phase A and B. When the proposed function works, the grid-connected current is changed, shown as Fig. 14(b). The current in phase $\mathrm{C}$ is larger than the current in other two phase, in order to keep the active power unchanged.

As the ripples account for less percentage in the DC voltage, the difference values between the reference value $150 \mathrm{~V}$ and the actual value is shown in Fig.15. $U_{d c l}$ ' is the difference value before the proposed function works, which 
is saved in the oscilloscope. The ripples are nearly up to $3 \mathrm{~V}$. $U_{d c 2}$ ' is the voltage fluctuation after suppression which is reduced to $1.5 \mathrm{~V}$. MMC is proved to have the single phase to ground fault-tolerance capability with the proposed control strategy.

\section{Conclusion}

In this paper, OCC is applied in the control of MMC according to the volt-second equilibrium. An improved structure is introduced based on the basic strategy when single phase to ground fault happens. The virtual loop mapping is also introduced in the paper. The conclusion of the whole paper is as

(1) MMC control strategy based on OCC has the obvious advantages of simple structure, stability and rapid response.

(2) When single phase to ground fault happens, the ripples in the DC side is suppressed by the improved control strategy based on the condition of constant power. In the simulation and the experiment, the ripples decrease from $2 \%$ to $1 \%$ with no need for measuring the positive and negative current sequence.

(3) The capacitor voltage of SM is balanced with the introduction of the virtual loop mapping. With no need to measure SM, the voltage is held to $37 \mathrm{~V}$ in the simulation and experiment.

(4) An improved OCC control structure is proposed in the paper. With no effect to the control signals, the structure is simplified.

\section{References}

[1] Rodriguez, J.; Jih-Sheng Lai; Fang Zheng Peng, "Multilevel inverters: a survey of topologies, controls, and applications," in Industrial Electronics, IEEE Transactions on, vol.49, no.4, pp.724-738, Aug 2002

[2] Allebrod, S.; Hamerski, R.; Marquardt, R., "New transformerless, scalable Modular Multilevel Converters for HVDC-transmission," in Power Electronics Specialists Conference, 2008. PESC 2008. IEEE, vol., no., pp.174-179, 15-19 June 2008

[3] Malinowski, M.; Gopakumar, K.; Rodriguez, J.; Pérez, M.A., "A Survey on Cascaded Multilevel Inverters," in Industrial Electronics, IEEE Transactions on, vol.57, no.7, pp.2197-2206, July 2010

[4] Rohner, S.; Bernet, S.; Hiller, M.; Sommer, R., "Modulation, Losses, and Semiconductor Requirements of Modular Multilevel Converters," in Industrial Electronics, IEEE Transactions on, vol. 57, no. 8, pp. 2633-2642, Aug. 2010

[5] Solas, E.; Abad, G.; Barrena, J.A.; Aurtenetxea, S.; Carcar, A.; Zajac, L., "Modular Multilevel Converter
With Different Submodule Concepts - Part II: Experimental Validation and Comparison for HVDC Application," in Industrial Electronics, IEEE Transactions on, vol. 60, no. 10, pp. 4536-4545, Oct. 2013

[6] Lesnicar, A.; Marquardt, R., "An innovative modular multilevel converter topology suitable for a wide power range," in Power Tech Conference Proceedings, 2003 IEEE Bologna, vol.3, no., pp. 6, Vol. 3-, 23-26 June 2003

[7] Hagiwara, M.; Akagi, H., "Control and Experiment of Pulsewidth-Modulated Modular Multilevel Converters," in Power Electronics, IEEE Transactions on, vol.24, no.7, pp.1737-1746, July 2009

[8] Rohner, S.; Bernet, S.; Hiller, M.; Sommer, R., "Modulation, Losses, and Semiconductor Requirements of Modular Multilevel Converters," in Industrial Electronics, IEEE Transactions on, vol. 57, no. 8, pp. 2633-2642, Aug. 2010

[9] Ilves, K.; Antonopoulos, A.; Norrga, Staffan; Nee, H.-P., "A New Modulation Method for the Modular Multilevel Converter Allowing Fundamental Switching Frequency," in Power Electronics, IEEE Transactions on, vol. 27, no. 8, pp. 3482-3494, Aug. 2012

[10] Gemmell, B.; Dorn, J.; Retzmann, D.; Soerangr, D., "Prospects of multilevel VSC technologies for power transmission," in Transmission and Distribution Conference and Exposition, 2008. T\&D. IEEE/PES, vol., no., pp.1-16, 21-24 April 2008

[11] Adam, G. P.; Anaya-Lara, O.; Burt, G. M.; Telford, D.; Williams, B. W.; McDonald, J. R., "Modular multilevel inverter: Pulse width modulation and capacitor balancing technique," in Power Electronics, IET, vol.3, no.5, pp.702-715, September 2010

[12] Hagiwara, M.; Akagi, H., "Control and Experiment of Pulsewidth-Modulated Modular Multilevel Converters," in Power Electronics, IEEE Transactions on, vol.24, no.7, pp.1737-1746, July 2009

[13] Kui Wang; Yongdong Li; Zedong Zheng; Lie Xu, "Voltage Balancing and Fluctuation-Suppression Methods of Floating Capacitors in a New Modular Multilevel Converter," in Industrial Electronics, IEEE Transactions on, vol. 60, no. 5, pp. 1943-1954, May 2013

[14] Ng, Chong; Ran, L.; Bumby, J., "Unbalanced Grid Fault Ride-Through Control for a Wind Turbine Inverter," in Industry Applications Conference, 2007. 42nd IAS Annual Meeting. Conference Record of the 2007 IEEE, vol. no., pp. 154-164, 23-27 Sept. 2007

[15] Saeedifard, M.; Iravani, R., "Dynamic Performance of a Modular Multilevel Back-to-Back HVDC System," in Power Delivery, IEEE Transactions on, vol. 25, no. 4, pp. 2903-2912, Oct. 2010

[16] Minyuan Guan; Zheng Xu, "Modeling and Control of a Modular Multilevel Converter-Based HVDC System Under Unbalanced Grid Conditions," in 
Power Electronics, IEEE Transactions on, vol. 27, no. 12, pp. 4858-4867, Dec. 2012

[17] Yuebin Zhou; Daozhuo Jiang; Jie Guo; Pengfei Hu; Yiqiao Liang, "Analysis and Control of Modular Multilevel Converters Under Unbalanced Conditions," in Power Delivery, IEEE Transactions on, vol. 28, no. 4, pp. 1986-1995, Oct. 2013

[18] Xiaojie Shi; Zhiqiang Wang; Bo Liu; Yiqi Liu; Tolbert, L.M.; Wang, F., "Characteristic Investigation and Control of a Modular Multilevel ConverterBased HVDC System Under Single-Line-to-Ground Fault Conditions," in Power Electronics, IEEE Transactions on, vol. 30, no. 1, pp. 408-421, Jan. 2015

[19] Li Shao-long; Jin Ai-juan; Li Hang-tian, "An Improved and Digital Current Control Strategy for One Cycle Control Based Three-phase Boost Rectifier under Unbalanced System," in Power Electronics and Motion Control Conference, 2006. IPEMC 2006. CES/IEEE 5th International, vol.2, no., pp. 1-5, 1416 Aug. 2006

[20] K. Smedley and S. Ćuk, "Dynamics of one-cycle controlled Cuk converters," in IEEE Transactions on Power Electronics, vol. 10, no. 6, pp. 634-639, Nov 1995.

[21] C. C. Chan, Zheng Ming Zhao, C. Qian and S. Meng, "Comparisons of PWM and one-cycle control for power amplifier with multilevel converter, " in IEEE Transactions on Industrial Electronics, vol. 49, no. 6, pp. 1342-1344, Dec 2002.

[22] Chongming Qiao, Taotao Jin and K. M. Smedley, "One-cycle control of three-phase active power filter with vector operation," in IEEE Transactions on Industrial Electronics, vol. 51, no. 2, pp. 455-463, April 2004.

[23] D. Yang, M. Yang and X. Ruan, "One-Cycle Control for a Double-Input DC/DC Converter," in IEEE Transactions on Power Electronics, vol. 27, no. 11, pp. 4646-4655, Nov. 2012.

[24] Qingrui Tu; Zheng Xu; Lie Xu, "Reduced SwitchingFrequency Modulation and Circulating Current Suppression for Modular Multilevel Converters," in Power Delivery, IEEE Transactions on, vol. 26, no. 3, pp. 2009-2017, July 2011

[25] Chongming Qiao; Smedley, K.M., "A general threephase PFC controller. I. For rectifiers with a parallelconnected dual boost topology," in Industry Applications Conference, 1999. Thirty-Fourth IAS Annual Meeting. Conference Record of the 1999 IEEE, vol.4, no., pp.2504-2511 vol.4, 1999

[26] Zhou Lin; Zhou Xiaojun; Zhou Luowei; Heng-Ming, T., "One-cycle control for three-phase four-leg active power filter," in Industrial Electronics Society, 2004. IECON 2004. 30th Annual Conference of IEEE, vol.1, no., pp.558-563 Vol. 1, 2-6 Nov. 2004

[27] Stankovic, A.V.; Lipo, T.A., "A novel control method for input output harmonic elimination of the PWM boost type rectifier under unbalanced operating conditions," in Power Electronics, IEEE Transactions on, vol.16, no.5, pp.603-611, Sep 2001

[28] Jun Mei; Ke Shen; Bailu Xiao; Tolbert, L.M.; Jianyong Zheng, "A New Selective Loop Bias Mapping Phase Disposition PWM With Dynamic Voltage Balance Capability for Modular Multilevel Converter," in Industrial Electronics, IEEE Transactions on, vol. 61, no. 2, pp. 798-807, Feb. 2014

[29] Yang Chen; Smedley, K.M., "One-Cycle-Controlled Three-Phase Grid-Connected Inverters and Their Parallel Operation," in Industry Applications, IEEE Transactions on, vol. 44, no. 2, pp. 663-671, Marchapril 2008

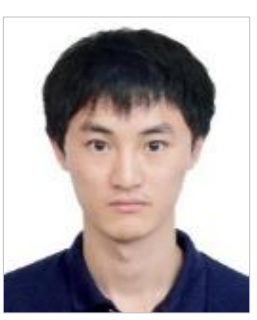

Huiyu Miao was born in Jiangsu, China. He received his B.S. from Southeast University (SEU), Nanjing, China, in 2014, in Electrical Engineering. He is now pursing his Ph.D. in Electrical Engineering. His current research interests include the application of power electronics in power system and voltage sourceconverter-based HVDC..

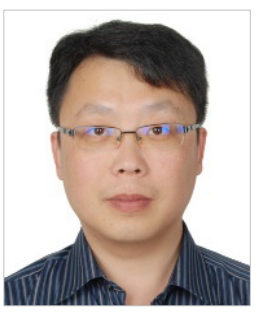

Jun Mei received the B.S. degree in radio engineering from the Chongqing University in 1994, and the M.S. and $\mathrm{Ph} . \mathrm{D}$. degrees in electrical engineering from Southeast University, Nanjing, China, in 2001 and 2006. He is now an associate professor in the School of Electrical Engineering, Southeast University. From 2011 to 2012, he was a visiting scholar in University of Tennessee, Knoxville, TN. His interests are electric power converters for distributed energy sources, FACTS and power quality control.

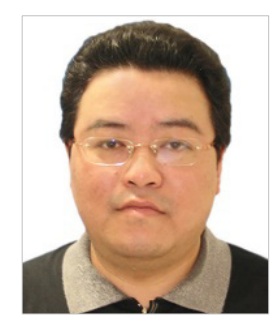

Jianyong Zheng was born in China, in 1966. He received his B.S., M.S., and Ph.D. in the School of Electrical Engineering from Southeast University, Nanjing, China in 1988, 1991, and 1999, respectively. He is now a Full Professor in the School of Electrical Engineering, Southeast University. His research interests are in the fields of the application of power electronics in power system and renewable energy technology. 


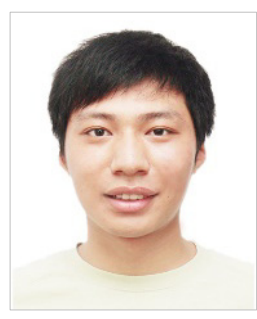

Chenyu Zhang was born in Jiangsu, China. He received his B.S. from Hunan University, Changsha, China, in 2011, and the Ph.D. degree in electrical engineering from Southeast University, Nanjing, China, in 2011 and 2016. He is now working. in Jiangsu Electric Power Research Institute, Nanjing, China. His current research interests include micro-grid power quality, harmonic suppression and digital control of power converters.

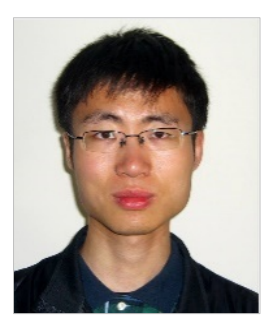

Chao Zhu was born in Jiangsu Nanjing, on January 12, 1987. He received the doctor's degree in Electrical Engineering Department from the Southeast University in 2014. And he work in the Jiangsu electric power maintenance branch company now. His research interests are smart grid, on-line monitoring technology and signal processing.

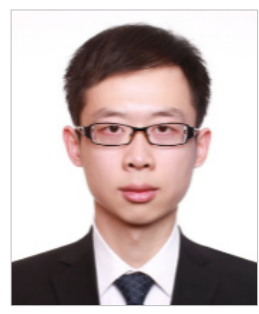

Tian Ma received the B.S. and M.S. degrees in electrical engineering from Southeast University, NanJing, China in 2013 and 2016, respectivley. He is now working. in State Grid Jiangsu Economic Research Institute, Nanjing, China. His current research interests include the application of power electronics in power system and voltage sourceconverter-based HVDC.

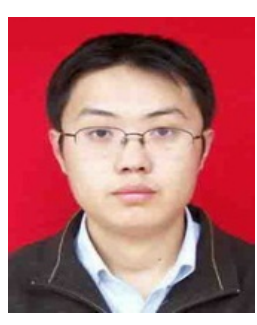

Fei Mei received the B.S. degree in mechanical engineering from SEU in 2002, master's degree in mechanical engineering from SEU in 2005 and Doctor Degree in the School of Electrical Engineering from SEU in 2014. He is a teacher at the College of Energy and Electrical Engineering in Hohai University now. His research interests are power electronics, smart grid, on-line monitoring technology and signal processing. 